\title{
Nano and Microscale Light-based 3D Bioprinting for Tissue Engineering and Regenerative Medicine
}

\author{
Shaochen Chen, Ph.D. \\ Professor and Chair of NanoEngineering Department \\ Professor of Bioengineering Department \\ Founding Co-Director, Biomaterials \& Tissue Engineering Center \\ University of California, San Diego
}

\begin{abstract}
In this talk, I will present our laboratory's recent research efforts in developing micro and nano-scale bioprinting methods to create 3D tissue constructs using a variety of biomaterials and cells. These 3D printed scaffolds are functionalized with precise control of micro-architecture, mechanical (e.g. stiffness), chemical, and biological properties. Such functional scaffolds allow us to investigate cell-microenvironment interactions at nano- and micro-scales in response to integrated mechanical and chemical stimuli. From these fundamental studies we have been creating both in vitro and in vivo precision tissues for tissue regeneration, disease modeling, and drug discovery. Examples including 3D bioprinted liver and heart models will be discussed. I will also showcase 3D printed biomimetic scaffolds for treating spinal cord injury. Throughout the presentation, I will discuss engineer's perspectives in terms of design innovation, biomaterials, mechanics, and scalable biomanufacturing.
\end{abstract}

